\title{
Diagnostic and prognostic implications of serum miR-101 in osteosarcoma
}

\author{
Z.-S. Yao ${ }^{\mathrm{a}}$, C. Li ${ }^{\mathrm{b}, *}$, D. Liang ${ }^{\mathrm{a}}$, X.-B. Jiang ${ }^{\mathrm{a}}$, J.-J. Tang ${ }^{\mathrm{a}}$, L.-Q. Ye ${ }^{\mathrm{a}}$, K. Yuan ${ }^{\mathrm{a}}$, H. Ren ${ }^{\mathrm{a}}$, Z.-D. Yang ${ }^{\mathrm{a}}$, \\ D.-X. Jin ${ }^{\mathrm{a}}$, S.-C. Zhang ${ }^{\mathrm{a}}$, J.-Y. Ding ${ }^{\mathrm{a}}$, Y.-C. Tang ${ }^{\mathrm{a}}$, J.-X. Xu ${ }^{\mathrm{a}}$, K. Chen ${ }^{\mathrm{a}}$, W.-X. Xie ${ }^{\mathrm{a}}$, D.-Q. Guo ${ }^{\mathrm{a}}$ and \\ J.-C. Cui ${ }^{\mathrm{a}}$ \\ ${ }^{a}$ Departments of Spinal Surgery, The First Affiliated Hospital of Guangzhou University of Traditional Chinese \\ Medicine, Guangzhou, Guangdong, China \\ ${ }^{\mathrm{b}}$ Departments of Clinical Laboratory, The First Affiliated Hospital of Guangzhou University of Traditional Chinese \\ Medicine, Guangzhou, Guangdong, China
}

\begin{abstract}
Blood-circulating microRNAs (miRNAs) have been reported to be used as potential biomarkers in various cancers. MiR-101 has been found to act as a tumor suppressor in many tumor types, but little is known for osteosarcoma. The purpose of this study was to investigate miR-101 expression in osteosarcoma patients and assess its correlation with clinical features and prognosis. Serum samples from 152 osteosarcoma patients and 70 healthy controls were detected using quantitative reversetranscription polymerase chain reaction (qRT-PCR). The data showed that miR-101 expression levels were remarkably underexpressed in serum samples from osteosarcoma patients compared to controls, and the post-treatment serum miR-101 expression was significantly higher than that in the pre-treatment expression. Low serum miR-101 expression was positively associated with advanced clinical stage and distant metastasis. Receiver operating characteristic (ROC) curve analysis showed that serum miR-101 could serve as a useful marker for osteosarcoma diagnosis, with a high sensitivity and specificity. Moreover, patients with high miR-101 expression had longer overall survival and recurrence free survival than those with low miR-101 expression. In addition, both univariate and multivariate analyses showed that serum miR-101 downregulation was associated with shorter overall survival and recurrence free survival. Our present results implicated serum miR-101 might be a useful biomarker for the clinical diagnosis and prognosis of osteosarcoma.
\end{abstract}

Keywords: MiR-101, serum, osteosarcoma, biomarker

\section{Introduction}

The lack of effective biomarkers is one of the most crucial problems for osteosarcoma, which is the most frequent type of bone malignancies and the leading cause of cancer related mortality in children and young adults $[1,2]$. Despite current advancements in diagnosis and therapy of osteosarcoma, the 5-year survival rate of this disease is still poor, and becomes unacceptably low for patients with recurrence or distant metastasis $[3,4]$. Thus, the identification of possible

\footnotetext{
${ }^{*}$ Corresponding author: C. Li, Department of Clinical Laboratory, The First Affiliated Hospital of Guangzhou University of Traditional Chinese Medicine, Guangzhou, Guangdong, China. Tel.: +86 20 36591912; E-mail: gzzyfy_cuili1990@163.com.
}

biomarker is urgently needed to improve the diagnosis and prognostic predictions of osteosarcoma.

MicroRNAs (miRNAs) are a class of conserved small non-coding RNAs, which regulate target gene expression at the post-transcriptional level [5,6]. Accumulated studies have demonstrated miRNAs play critical roles in cancer progression as either oncogenes or tumor suppressors [7]. During tumor initiation and progression, miRNAs are found to involve in diverse biological processes, such as cell proliferation, invasion, metastasis and angiogenesis [8,9]. Several miRNAs, such as miR-95-3p [10], miR-300 [11], miR-101, are found to be aberrantly expressed in osteosarcoma.

MiR-101 is encoded by a gene located at chromosome 1p31.3 and chromosome 9p24.1 [12]. Previous studies have reported that miR-101 might act as a tu- 
mor suppressor in osteosarcoma. For instance, miR101 levels were found to be significantly lower both in osteosarcoma bone tissues and cancer cell lines. Moreover, restoration of miR-101 significantly suppressed cancer cell viability, migration, invasion and induced apoptosis, while the inhibitory effect of miR101 was reversed by overexpression of ROCK1 [13] or c-FOS [14]. Similarly, in vitro data showed miR-101 greatly decreased chemotherapy-induced autophagy and autophagy-related proteins expression in osteosarcoma cells, and blocked autophagy by miR-101 enhanced the sensitivity of cancer cells to chemotherapy [15]. However, the diagnostic or prognostic value of serum miR-101 in osteosarcoma remains poor known.

The aim of our study was to explore the clinical significance of miR-101 in osteosarcoma. We detected serum miR-101 expression levels in all the participants, and evaluated the association between serum miR-101 expression and clinical features of patients with osteosarcoma. Moreover, the prognostic value of the miR-101 expression in osteosarcoma was analyzed with Kaplan-Meier method as well as univariate/multivariate analyses.

\section{Materials and methods}

\subsection{Ethical considerations}

Written informed consent was provided by each participant, and the protocols of our study were approved by the Ethics Committee of the First Affiliated Hospital of Guangzhou University of Traditional Chinese Medicine before commencement.

\subsection{Participants}

A total of 152 osteosarcoma patients and 70 healthy individuals were enrolled in the current study. The controls were matched to cases based on age and gender. None of the patients had received any treatment before surgery. Demographic information of 152 patients and detailed clinical data were collected and presented in Table 1. The diagnosis of osteosarcoma was based on image tests (X-ray, computerized tomography and magnetic resonance imaging) and biopsy. Osteosarcoma was classified according to World Health Organization classification system. Each patient received regular follow-up until death or last followup. The serum samples were collected from Depart-
Table 1

Association of serum miR-101 level with clinical characteristics of 152 osteosarcoma patients

\begin{tabular}{|c|c|c|c|c|}
\hline \multirow[t]{2}{*}{ Clinical features } & \multirow[t]{2}{*}{ Cases } & \multicolumn{2}{|c|}{ MiR-101 expression } & \multirow[t]{2}{*}{$P$ value } \\
\hline & & Low & High & \\
\hline Age & 152 & 82 & 70 & 0.449 \\
\hline$<55$ & 84 & 43 & 41 & \\
\hline$\geqslant 55$ & 68 & 39 & 29 & \\
\hline Sex & & & & 0.525 \\
\hline Men & 87 & 45 & 42 & \\
\hline Women & 65 & 37 & 28 & \\
\hline Clinical stage & & & & 0.013 \\
\hline IIA & 66 & 28 & 38 & \\
\hline IIB/III & 86 & 54 & 32 & \\
\hline Tumor diameter & & & & 0.167 \\
\hline$\leqslant 8 \mathrm{~cm}$ & 69 & 33 & 36 & \\
\hline$>8 \mathrm{~cm}$ & 83 & 49 & 34 & \\
\hline Distant metastasis & & & & 0.028 \\
\hline Positive & 21 & 16 & 5 & \\
\hline Negative & 131 & 66 & 65 & \\
\hline Anatomic location & & & & 0.084 \\
\hline Tibia/femur & 93 & 45 & 48 & \\
\hline Elsewhere & 59 & 37 & 22 & \\
\hline
\end{tabular}

Table 2

Primer sequences for real-time PCR analysis

\begin{tabular}{ll}
\hline Primers & Primer sequence (5'-3') \\
\hline $\begin{array}{c}\text { miR-101 } \\
\text { (forward) } \\
\text { miR-101 } \\
\quad \text { (reverse) }\end{array}$ & TATGAATTCATTCTAATTTAATTCAACTGG \\
U6 (forward) & CTCGCTTCGGCAGCACA \\
U6 (reverse) & AACGCTTCACGAATTTGCGT \\
\hline
\end{tabular}

ments of Clinical Laboratory, The First Affiliated Hospital of Guangzhou University of Traditional Chinese Medicine, and then placed in tubes and stored at $-80^{\circ} \mathrm{C}$ for RNA extraction.

\subsection{RNA isolation and quantitative RT-PCR}

Total RNA isolation was carried out using TaqMan miRNA Isolation Kit (TaKaRa, Otsu, Shiga, Japan). RNA purity and concentrations were assessed by a NanoDrop spectrophotometer (ND-1000, Thermo Scientific, Wilmington, USA). Reverse transcription was performed using TaqMan MicroRNA Reverse Transcription Kit (Applied Biosystems, Foster City, CA, USA). PCR using a Taqman MicroRNA Assay Kit (TaKaRa, Otsu, Shiga, Japan) on a MyiQ Real-Time PCR Detection System (Bio-Rad, Richmond, CA, USA). The PCR primers for miR-101 were presented in Table 2. U6 was used as an endogenous control, and the relative serum miR-101 expression levels were determined using the $2^{-\Delta \Delta \mathrm{Ct}}$ method. All reactions were run in triplicate. 


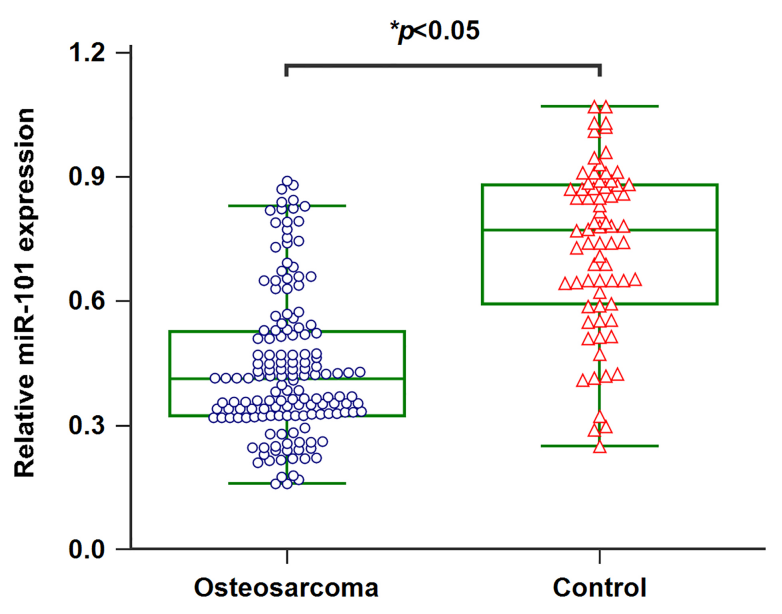

Fig. 1. Downregulation of miR-101 in serum of osteosarcoma patients.

\subsection{Statistical analysis}

Statistical analyses were performed using MedCalc 13.0 (MedCalc Software, Ostend, Belgium) and GraphPad Prism 6.04 (GraphPad Software, La Jolla, CA, USA). The difference in serum miR-101 levels between the patients and controls was compared with Mann Whitney $U$ test. The association between miR-101 expression and various clinicopathological characteristics was analyzed with Chi-square test. Receiver operating characteristic (ROC) curve was conducted to assess the efficacy of miR-101 in discriminating osteosarcoma patients from healthy subjects. Survival curves were constructed with the Kaplan-Meier method and compared by the log-rank tests. Cox proportional hazard models of factors associated with survival were used to evaluate risk ratios. A level of $P<$ 0.05 was considered statistically significant.

\section{Results}

\subsection{Serum miR-101 was decreased in osteosarcoma patients}

qRT-PCR was processed to detect the serum miR101 levels in 152 osteosarcoma patients as well as 70 healthy controls. Serum miR-101 levels in osteosarcoma cases were significantly lower than those in healthy controls (Fig. 1, $P<0.05$ ). ROC curve analysis indicated serum miR-101 levels yielded an AUC of 0.850 in differentiating osteosarcoma subjects from healthy volunteers, the sensitivity and specificity were $78.95 \%$ and $82.86 \%$, respectively (Fig. 2).

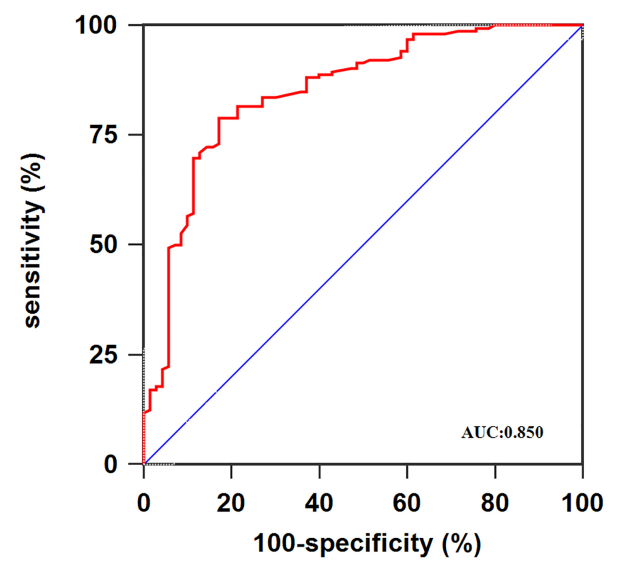

Fig. 2. ROC curve analysis using serum miR-101 for discriminating osteosarcoma patients.

\subsection{Alterations of serum miR-101 levels in osteosarcoma patients}

We then withdrawn the post-treatment blood specimens from all patients and investigated the alteration of serum miR-101 levels in paired pre-treatment samples. As shown in Fig. 3A, miR-101 levels were markedly elevated in post-treatment blood samples $(P<0.05)$. Likewise, the dynamic changes in serum miR-101 of patients without metastasis were analyzed, and a significant increase in miR-101 expression was observed in post-treatment blood samples (Fig. 3B, $P<0.01$ ). Whereas, no significant difference was found in miR101 expression prior or after surgery in patients with metastasis (Fig. 3C, $P>0.05$ ).

\subsection{Association of miR-101 expression with advanced clinicopathological characteristics of osteosarcoma patients}

The median miR-101 level was used as a cutoff value to divide 152 osteosarcoma cases into two groups, 82 cases showed low miR-101 expression while 70 subjects showed high miR-101 expression. As shown in Table 1, serum miR-101 was strongly correlated with clinical stage $(P=0.013)$ and distant metastasis $(P=0.028)$. In contrast, there was no association of serum miR-101 expression with age, sex, tumor diameter, anatomic location (all $P>0.05$ ).

\subsection{Association of miR-101 expression with clinical prognosis}

Furthermore, Kaplan-Meier survival analysis and 
Table 3

Univariate analysis of prognostic factors in 152 osteosarcoma patients

\begin{tabular}{|c|c|c|c|c|c|c|}
\hline \multirow[t]{2}{*}{ Variables } & \multicolumn{3}{|c|}{ Overall survival } & \multicolumn{3}{|c|}{ Recurrence free survival } \\
\hline & RR & $95 \% \mathrm{CI}$ & $P$ & RR & $95 \% \mathrm{CI}$ & $P$ \\
\hline Clinical stage (IIB/III vs. IIA) & 3.42 & $1.21-5.88$ & 0.019 & 3.14 & $1.09-5.32$ & 0.025 \\
\hline Distant metastasis (Positive vs. Negative) & 3.25 & $1.16-5.45$ & 0.023 & 3.72 & $1.33-6.26$ & 0.017 \\
\hline MiR-101 expression (Low vs. high) & 4.17 & $1.43-7.08$ & 0.012 & 4.45 & $1.57-7.49$ & 0.006 \\
\hline
\end{tabular}

${ }^{*} P \leqslant 0.05$. CI, confidence interval; RR, risk ratio.
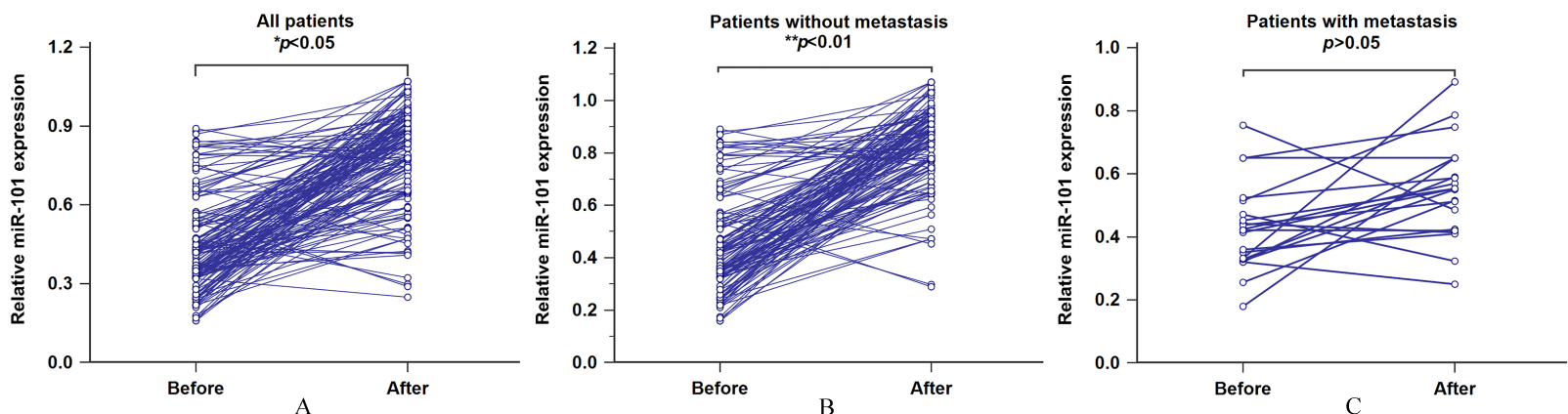

Fig. 3. A. The association between serum miR-101 levels and therapeutic response for all patients. B. The association between serum miR-101 levels and therapeutic response for the patients without metastasis. C. The association between serum miR-101 levels and therapeutic response for the patients with metastasis.

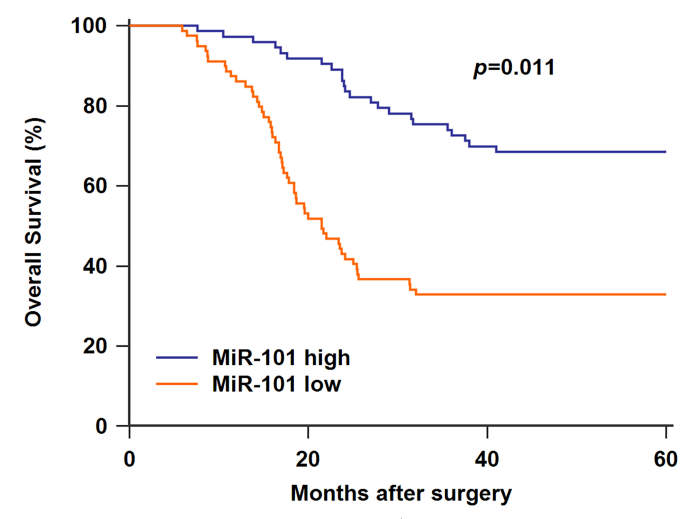

A

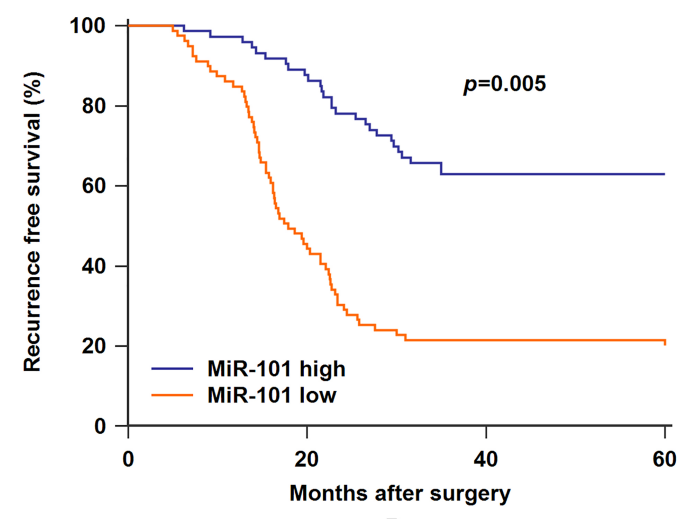

Fig. 4. A. Low serum miR-101 expression was associated with worse overall survival. B. Low serum miR-101 expression was associated with worse recurrence free survival.

log-rank test were performed to further reveal the prognosis of osteosarcoma patients. The patients with low miR-101 expression suffered worse overall survival (OS) and recurrence free survival (RFS) compared to those exhibiting high miR-101 expression (Fig. 4A, $P=0.011$; Fig. 4B, $P=0.005)$. Univariate analysis confirmed low miR-101 expression $(P=0.012$; $P=0.006)$, distant metastasis $(P=0.023 ; P=$ $0.017)$ and clinical stage $(P=0.019 ; P=0.025)$ as poor prognostic factors for OS/RFS (Table 3). Multivariate analysis demonstrated low miR-101 expression $(P=0.014 ; P=0.008)$, distant metastasis $(P=$ $0.027 ; P=0.020)$ and clinical stage $(P=0.025$;
$P=0.018)$ were independent prognostic indicators of OS/RFS (Table 4).

\section{Discussion}

Since miRNAs are highly stable and easily detected in blood/serum, many serum miRNAs are identified as novel non-invasive biomarkers for cancer diagnosis and prognosis. In terms of osteosarcoma, a number of circulating miRNAs have been reported to be underexpressed or overexpressed. For example, downregulation of miR-95-3p was found in osteosarcoma patients 
Table 4

Multivariate analysis of prognostic factors in 152 osteosarcoma patients

\begin{tabular}{|c|c|c|c|c|c|c|}
\hline \multirow[t]{2}{*}{ Variables } & \multicolumn{3}{|c|}{ Overall survival } & \multicolumn{3}{|c|}{ Recurrence free survival } \\
\hline & RR & $95 \% \mathrm{CI}$ & $P$ & RR & $95 \% \mathrm{CI}$ & $P$ \\
\hline Clinical stage (IIB/III vs. IIA) & 3.15 & $1.16-5.24$ & 0.025 & 3.46 & $1.21-5.78$ & 0.018 \\
\hline Distant metastasis (Positive vs. Negative) & 2.94 & $1.02-4.98$ & 0.027 & 3.36 & $1.14-5.71$ & 0.020 \\
\hline MiR-101 expression (Low vs. high) & 3.82 & $1.34-6.52$ & 0.014 & 4.23 & $1.45-7.22$ & 0.008 \\
\hline
\end{tabular}

${ }^{*} P \leqslant 0.05$. CI, confidence interval; RR, risk ratio.

compared to healthy controls, and low miR-95-3p expression was positively correlated with clinical stage and metastasis, as well as shorter survival and diseasefree survival, indicating miR-95-3p served as a tumor suppressor in osteosarcoma [10]. Conversely, Liu et al. showed miR-300 levels in osteosarcoma cases were significantly higher than those in healthy controls, and aberrantly elevated miR-300 expression was associated with worse prognosis of patients, suggesting an oncogenic role of miR-300 [11].

Up to now, extensive researches have demonstrated that altered expression of miR-101 contributed to tumorigenesis in multiple types of human cancers. In lung cancer, the levels of DNMT3A and miR-101 were negatively correlated in cancer cell lines or clinical tissues, and either DNMT3A overexpression or loss of miR-101 enhanced cell proliferation, migration in vitro and promoted tumorigenicity in vivo $[16,17]$. Another study by Cho et al. found loss of EZH2 suppressed the carcinogenesis of lung cancer, while miR101 downregulation greatly attenuated the inhibitory effect [18]. In breast cancer, miR-101 was inversely correlated with aggressive progression, and its restoration remarkably restrained carcinogenesis in vitro and in vivo through the repression of SOX-2 [19], or CXCR7 [20]. Furthermore, it was reported that miR101 was markedly downregulated in papillary thyroid carcinoma tissues, and attenuated tumor growth in vitro by targeting Rac1 [21] or USP22 [22]. Moreover, miR-101 was dramatically underexpressed in hepatocellular carcinoma tissues, and forced miR-101 expression reduced the oncogenic activities of cancer cells. Moreover, VEGF-C [23] and Girdin [24] were identified as its downstream target. In esophageal squamous cell carcinoma, miR-101 downregulation was observed both in cancerous tissues and cell lines. MiR101 overexpression significantly inhibited cancer proliferation and motility through directly silencing COX2 [25], or EZH2 [26]. In ovarian cancer, the proliferation, migration and invasion capacity of cancer cells was dramatically reduced by miR-101 upregulation, and miR-101 expression was inversely associated with brain-derived neurotrophic factor [27], SOCS-
2 [28], ZEB1 and ZEB2 [29]. In glioblastoma multiforme (GBM), decreased miR-101 expression have been found in GBM relative to normal samples, and in vitro and in vivo evidence demonstrated elevated miR-101 expression was strongly associated with reduced cell growth and motility [30,31]. Lin et al. reported miR-101 upregulation was mostly correlated to significant decreased cellular motility of cervical cancer [32]. Also, miR-101 downregulation was found in patients with cervical lymph node metastasis and associated with shortened survival time [33]. In bladder transitional cell carcinoma, low miR-101 expression predicted worse prognosis of patients, and had strong association with the presence of advanced tumor stage and lymph node metastasis [34]. Additionally, He and his colleagues demonstrated miR-101 levels were decreased in gastric cancer tissues compared to normal paracancerous tissues, low miR-101 expression led to increased cancer cell proliferation and reduced cell apoptosis by inducing expression of COX2 [35]. In prostate cancer, Hao et al. provided in vitro and in vivo analysis to reveal ectopic miR-101 expression markedly inhibited the tumorigenic properties of cancer cells, with COX-2 as a direct target [36].

The results from this exploratory study revealed for the first time that the miR-101 expression was dramatically decreased in the serum of osteosarcoma patients. Then, the expression of miR-101 showed a moderate diagnostic value for osteosarcoma (AUC = 0.850 ), with a sensitivity of $78.95 \%$ and specificity of $82.86 \%$. Furthermore, decreased miR-101 expression was found to be strongly linked to higher clinical stage and distant metastasis. For osteosarcoma patients without distant metastasis, the levels of miR101 in the post-treatment blood samples were higher than those in the pre-treatment samples, suggesting that serum miR-101 levels might be used for monitoring the therapeutic responses. Kaplan-Meier survival analysis demonstrated that patients with high miR-101 expression had significantly longer OS/RFS. Notably, serum miR-101, clinical stage and distant metastasis retained significance as independent prognostic indicators of OS/RFS of osteosarcoma patients. Our results 
were consistent with previous reports [13-15]. The possible reasons accounting for decreased serum miR101 in patients with osteosarcoma might be as follows: 1. The level of miR-101 is reduced in the tumor cells, leading to less miR-101 secretion into the circulation system; 2. Exosomes are circulating extracellular vesicles in the biofluids carrying various functional components including DNA, proteins, lipids, RNA, noncoding RNA and microRNA. Therefore, less miR-101 is packaged into the exosomes in cancer cells, resulting in reduced circulating miR-101 levels indirectly. Though our findings were promising, the limitation of the study was the small sample size. Thus, further investigations in a larger number of clinical samples are required.

In summary, the current study provides convincing evidence that miR-101 downregulation is a frequent event in osteosarcoma patients, and miR-101 may be used as a biomarker for the diagnosis and prognosis of this disease.

\section{Conflict of interest}

None.

\section{References}

[1] M. Kansara, M.W. Teng, M.J. Smyth and D.M. Thomas, Translational biology of osteosarcoma, Nat Rev Cancer 14(11) (2014), 722-735.

[2] D.M. Gianferante, L. Mirabello and S.A. Savage, Germline and somatic genetics of osteosarcoma-connecting aetiology, biology and therapy, Nat Rev Endocrinol 13(8) (2017), 480491.

[3] S. Wang, S. Zheng, K. Hu, H. Sun, J. Zhang, G. Rong, J. Gao, N. Ding and B. Gui, A predictive model to estimate the pretest probability of metastasis in patients with osteosarcoma, Medicine (Baltimore) 96(3) (2017), e5909.

[4] B.A. Lindsey, J.E. Markel and E.S. Kleinerman, Osteosarcoma overview, Rheumatol Ther 4(1) (2017), 25-43.

[5] D.P. Bartel, MicroRNAs: genomics, biogenesis, mechanism, and function, Cell 116 (2004), 281-297.

[6] W. Filipowicz, S.N. Bhattacharyya and N. Sonenberg, Mechanisms of post-transcriptional regulation by microRNAs: are the answers in sight? Nat Rev Genet 9 (2008), 102-114.

[7] B.M. Ryan, A.I. Robles and C.C. Harris, Genetic variation in microRNA networks: the implications for cancer research, Nat Rev Cancer 10 (2010), 389-402.

[8] Z. Li, X. Yu, J. Shen, W.K. Wu and M.T. Chan, MicroRNA expression and its clinical implications in Ewing's sarcoma, Cell Prolif 48(1) (2015), 1-6.

[9] A.E. Erson and E.M. Petty, MicroRNAs in development and disease, Clin Genet 74 (2008), 296-306.

[10] J. Niu, Y. Sun, Q. Guo, D. Niu and B. Liu, Serum miR-95$3 \mathrm{p}$ is a diagnostic and prognostic marker for osteosarcoma, Springerplus 5(1) (2016), 1947.
[11] J.D. Liu, Q. Xin, C.S. Tao, P.F. Sun, P. Xu, B. Wu, L. Qu and S.Z. Li, Serum miR-300 as a diagnostic and prognostic biomarker in osteosarcoma, Oncol Lett 12(5) (2016), 3912 3918 .

[12] T. Gui and K. Shen, MiRNA-101: a potential target for tumor therapy, Cancer Epidemiol 36(6) (2012), 537-540.

[13] R. Jiang, C. Zhang, G. Liu, R. Gu and H. Wu, MicroRNA-101 inhibits proliferation, migration and invasion in osteosarcoma cells by targeting ROCK1, Am J Cancer Res 7(1) (2017), 8897.

[14] Z. Wang, R. He, H. Xia, Y.U. Wei and S. Wu, MicroRNA101 has a suppressive role in osteosarcoma cells through the targeting of c-FOS, Exp Ther Med 11(4) (2016), 1293-1299.

[15] Z. Chang, L. Huo, K. Li, Y. Wu and Z. Hu, Blocked autophagy by miR-101 enhances osteosarcoma cell chemosensitivity in vitro, Scientific World Journal 2014 (2014), 794756.

[16] L. Wang, J. Yao, H. Sun, K. He, D. Tong, T. Song and C. Huang, MicroRNA-101 suppresses progression of lung cancer through the PTEN/AKT signaling pathway by targeting DNA methyltransferase 3A, Oncol Lett 13(1) (2017), 329338.

[17] F. Yan, N. Shen, J. Pang, D. Xie, B. Deng, J.R. Molina, P. Yang and S. Liu, Restoration of miR-101 suppresses lung tumorigenesis through inhibition of DNMT3a-dependent DNA methylation, Cell Death Dis 5 (2014), e1413.

[18] H.M. Cho, H.S. Jeon, S.Y. Lee, K.J. Jeong, S.Y. Park, H.Y. Lee, J.U. Lee, J.H. Kim, S.J. Kwon, E. Choi, M.J. Na, J. Kang and J.W. Son, MicroRNA-101 inhibits lung cancer invasion through the regulation of enhancer of zeste homolog 2, Exp Ther Med 2(5) (2011), 963-967.

[19] J. Wang, H. Zeng, H. Li, T. Chen, L. Wang, K. Zhang, J. Chen, R. Wang, Q. Li and S. Wang, MicroRNA-101 inhibits growth, proliferation and migration and induces apoptosis of breast cancer cells by targeting sex-determining region Y-Box 2, Cell Physiol Biochem 43(2) (2017), 717-732.

[20] J.T. Li, L.T. Jia, N.N. Liu, X.S. Zhu, Q.Q. Liu, X.L. Wang, F. Yu, Y.L. Liu, A.G. Yang and C.F. Gao, MiRNA-101 inhibits breast cancer growth and metastasis by targeting CX chemokine receptor 7, Oncotarget 6(31) (2015), 3081830830.

[21] X. Lin, H. Guan, H. Li, L. Liu, J. Liu, G. Wei, Z. Huang, Z. Liao and Y. Li, MiR-101 inhibits cell proliferation by targeting Rac1 in papillary thyroid carcinoma, Biomed Rep 2(1) (2014), 122-126.

[22] H. Zhao, H. Tang, Q. Huang, B. Qiu, X. Liu, D. Fan, L. Gong, H. Guo, C. Chen, S. Lei, L. Yang, J. Lu and G. Bao, MiR-101 targets USP22 to inhibit the tumorigenesis of papillary thyroid carcinoma, Am J Cancer Res 6(11) (2016), 2575-2586.

[23] Z. Liu, J. Wang, Y. Mao, B. Zou and X. Fan, MicroRNA101 suppresses migration and invasion via targeting vascular endothelial growth factor-C in hepatocellular carcinoma cells, Oncol Lett 11(1) (2016), 433-438.

[24] K. Cao, J. Li, Y. Zhao, Q. Wang, Q. Zeng, S. He, L. Yu, J. Zhou and P. Cao, MiR-101 inhibiting cell proliferation, migration and invasion in hepatocellular carcinoma through downregulating girdin, Mol Cells 39(2) (2016), 96-102.

[25] Y. Shao, P. Li, S.T. Zhu, J.P. Yue, X.J. Ji, Z. He, D. Ma L. Wang, Y.J. Wang, Y. Zong, Y.D. Wu and S.T. Zhang, Cyclooxygenase-2, a potential therapeutic target, is regulated by miR-101 in Esophageal squamous cell carcinoma, PLoS One 10(11) (2015), e0140642.

[26] C. Lin, F. Huang, Q.Z. Li and Y.J. Zhang, MiR-101 suppresses tumor proliferation and migration, and induces apoptosis by targeting EZH2 in esophageal cancer cells, Int J Clin Exp 
Pathol 7(10) (2014), 6543-6550.

[27] Y. Xu, L. Xu, J. Zheng, L. Geng and S. Zhao, MiR-101 inhibits ovarian carcinogenesis by repressing the expression of brain-derived neurotrophic factor, FEBS Open Bio 7(9) (2017), 1258-1266.

[28] H.B. Zheng, X.G. Zheng and B.P. Liu, MiRNA-101 inhibits ovarian cancer cells proliferation and invasion by downregulating expression of SOCS-2, Int J Clin Exp Med 8(11) (2015), 20263-20270.

[29] F. Guo, D. Cogdell, L. Hu, D. Yang, A.K. Sood, F. Xue and W. Zhang, MiR-101 suppresses the epithelial-to-mesenchymal transition by targeting ZEB1 and ZEB2 in ovarian carcinoma, Oncol Rep 31(5) (2014), 2021-2028.

[30] N. Liu, L. Zhang, Z. Wang, Y. Cheng, P. Zhang, X. Wang, W. Wen, H. Yang, H. Liu, W. Jin, Y. Zhang and Y. Tu, MicroRNA-101 inhibits proliferation, migration and invasion of human glioblastoma by targeting SOX9, Oncotarget $\mathbf{8}(12)$ (2017), 19244-19254.

[31] M. Smits, J. Nilsson, S.E. Mir, P.M. van der Stoop, E. Hulleman, J.M. Niers, P.C. de Witt Hamer, V.E. Marquez, J. Cloos, A.M. Krichevsky, D.P. Noske, B.A. Tannous and T. Würdinger, MiR-101 is down-regulated in glioblastoma resulting in EZH2-induced proliferation, migration, and angiogenesis, Oncotarget 1(8) (2010), 710-720.
[32] C. Lin, F. Huang, G. Shen and A. Yiming, MicroRNA-101 regulates the viability and invasion of cervical cancer cells, Int J Clin Exp Pathol 8(9) (2015), 10148-10155.

[33] W. Jiang, J.J. Pan, Y.H. Deng, M.R. Liang and L.H. Yao Down-regulated serum microRNA-101 is associated with aggressive progression and poor prognosis of cervical cancer, $J$ Gynecol Oncol 28(6) (2017), e75.

[34] H. Zhang, F. Qi, Y. Cao, M. Chen and X. Zu, Down-regulated microRNA-101 in bladder transitional cell carcinoma is associated with poor prognosis, Med Sci Monit 20 (2014), 812 817.

[35] X.P. He, Y. Shao, X.L. Li, W. Xu, G.S. Chen, H.H. Sun, H.C. Xu, X. Xu, D. Tang, X.F. Zheng, Y.P. Xue, G.C. Huang and W.H. Sun, Downregulation of miR-101 in gastric cancer correlates with cyclooxygenase-2 overexpression and tumor growth, FEBS J 279(22) (2012), 4201-4212.

[36] Y. Hao, X. Gu, Y. Zhao, S. Greene, W. Sha, D.T. Smoot, J. Califano, T.C. Wu and X. Pang, Enforced expression of miR101 inhibits prostate cancer cell growth by modulating the COX-2 pathway in vivo, Cancer Prev Res (Phila) 4(7) (2011), 1073-1083. 